\title{
Electrically Guiding Migration of Human Induced Pluripotent Stem Cells
}

\author{
Jiaping Zhang • Marco Calafiore • Qunli Zeng • \\ Xiuzhen Zhang • Yuesheng Huang • Ronald A. Li • \\ Wenbin Deng $\cdot$ Min Zhao
}

Published online: 5 March 2011

(C) The Author(s) 2011. This article is published with open access at Springerlink.com

\begin{abstract}
A major road-block in stem cell therapy is the poor homing and integration of transplanted stem cells with the targeted host tissue. Human induced pluripotent stem (hiPS) cells are considered an excellent alternative to embryonic stem (ES) cells and we tested the feasibility of using small, physiological electric fields (EFs) to guide hiPS cells to their target. Applied EFs stimulated and guided migration of cultured hiPS cells toward the anode, with a stimulation threshold of $<30 \mathrm{mV} / \mathrm{mm}$; in three-dimensional (3D) culture hiPS cells remained stationary, whereas in an applied EF they migrated directionally. This is of significance as the therapeutic use of hiPS cells occurs in a 3D environment. EF exposure did not alter expression of the pluripotency markers SSEA-4 and Oct-4 in hiPS cells. We compared EF-directed migration (galvanotaxis) of hiPS cells and hES cells and found that hiPS
\end{abstract}

Electronic supplementary material The online version of this article (doi:10.1007/s12015-011-9247-5) contains supplementary material, which is available to authorized users.

J. Zhang $\cdot$ Q. Zeng $\cdot$ X. Zhang $\cdot$ M. Zhao

Department of Dermatology and Department of Ophthalmology, Institute for Regenerative Cures, UC Davis School of Medicine,

Davis, CA 95817, USA

\section{J. Zhang $\cdot$ Y. Huang}

State Key Laboratory of Trauma,

Burns and Combined Injury,

Institute of Burns, Southwest Hospital,

Third Military Medical University,

Chongqing 400038, People's Republic of China

J. Zhang $\cdot$ M. Zhao

Center for Neuroscience, UC Davis School of Medicine,

Davis, CA 95618, USA

M. Calafiore $\cdot$ R. A. Li $\cdot$ W. Deng

Department of Cell Biology and Human Anatomy,

UC Davis School of Medicine,

Davis, CA 95817, USA cells showed greater sensitivity and directedness than those of hES cells in an EF, while hES cells migrated toward cathode. Rho-kinase (ROCK) inhibition, a method to aid expansion and survival of stem cells, significantly increased the motility, but reduced directionality of iPS cells in an EF by $70-80 \%$. Thus, our study has revealed that physiological EF is an effective guidance cue for the migration of hiPS cells in either $2 \mathrm{D}$ or $3 \mathrm{D}$ environments and that will occur in a ROCKdependent manner. Our current finding may lead to techniques for applying EFs in vivo to guide migration of transplanted stem cells.

Keywords Human induced pluripotent stem (hiPS) cell . Electric field (EF) · Cell migration · Wound healing ·

Galvanotaxis

Q. Zeng

Bioelectromagnetics Laboratory,

Zhejiang University School of Medicine,

Hangzhou 310058, People's Republic of China

X. Zhang

School of life Sciences, Shandong University of Technology,

Zibo 255049, People's Republic of China

M. Zhao $(\square)$

Institute for Regenerative Cures, UC Davis School of Medicine,

Suite 1600, 2921 Stockton Blvd,

Sacramento, CA 95817, USA

e-mail: minzhao@ucdavis.edu

W. Deng $(\square)$

Institute for Pediatric Regenerative Medicine,

Room 653, 2425 Stockton Blvd,

Sacramento, CA 95817, USA

e-mail: wbdeng@ucdavis.edu 


\section{Introduction}

Human induced pluripotent stem (hiPS) cells show great potential for stem cell based therapies, as they are very similar to human embryonic stem (hES) cells in their ability to self-renew and to differentiate into many types of cell within the body, and are free from the ethical issues to immunological barriers associated with the use of hES cells [1]. While there has been a great deal of interest in the pluripotency and differentiation of hiPS cells, little is known about their migration and guidance cues.

Amongst the various challenging hurdles to successful stem cell therapy, is the poor homing to and integration of transplanted stem cells with target tissue. Poor healing responses and lack of regeneration may be partly due to the fact that very few therapeutic stem cells actually migrate to the damaged/diseased tissue to mount a reparative response $[2,3]$. One example of such poor homing responses comes from a model of renal ischemia/reperfusion injury, in which intravenously injected mesenchymal stem cells (MSCs) sequestered mainly in the lung and liver, with less than $1 \%$ of injected MSCs reaching the injured kidney [4]. Also, homing of MSCs to infracted areas in heart has been shown to be very poor, and this contributes to poor survival rate [5]. Effective techniques to stimulate and guide migration of stem cells may therefore offer new strategies to improve the efficiency of stem cell therapies.

Signals that guide and regulate the migration of stem cells include growth factors (GFs), chemokines, molecules of the extracellular matrix (ECM), and their respective receptors. The importance of stromal-derived factor-1 (SDF-1), a well studied chemokine expressed by a variety of cell types including stromal cells and stem cells, and its unique receptor CXCR4 in migration and homing of stem cells has been demonstrated. This SDF-1/CXCR4 signaling plays a critical role in the homing of hematopoietic stem cells [6], the directional migration of neural stem cells to sites of central nervous system (CNS) injury [7], and the directional migration of endothelial progenitor cells [8]. In addition to SDF-1/CXCR4, the flucosylated CD44 has been shown to promote efficient adhesive interactions of MSCs with marrow vasculature and subsequent homing to endosteal surfaces [9]. Also, it has recently been suggested that insulin-like growth factor 2 (IGF-2)/IGF-2 receptor signaling is essential for homing of endothelial progenitor cells and vasculogenesis at ischemic or tumour sites [10]. In contrast, CD26 has been shown to be a negative regulator of hematopoietic stem cell homing [11].

Applied electric fields (EFs) may be another effective means to guide directional migration of hiPS cells. Damaged tissue and wounds produce weak, direct current (DC) EFs, which direct migration of many cell types. This phenomenon is known as galvanotaxis or electrotaxis [12].
With the aim of developing novel techniques to guide the migration of stem cells, we tested whether EFs direct the migration of hiPS cells. We found that: 1) applied EFs guide migration of isolated or clustered hiPS cells toward the anode, with a threshold voltage of $<30 \mathrm{mV} / \mathrm{mm}$; 2) EFs significantly increase migration speed of hiPS cells by $20 \%$ at $50 \mathrm{mV} / \mathrm{mm}$, and $40 \%$ at $75 \mathrm{mV} / \mathrm{mm}$; 3) EFs directed migration of hiPS cells in three-dimensional (3D) culture; 4) exposure to an EF did not alter expression of the stem cell markers SSEA-4 and Oct-4 in hiPS cells; 5) galvanotaxis of hiPS cells differed from that of hES cells in migratory speed, time-course of migration, to direction of migration; 6) Rho kinase (ROCK) inhibition significantly inhibited the directedness of migration, but increased the speed of migration in hiPS cells.

\section{Materials and Methods}

\section{Cell Culture}

Human iPS cells (foreskin-derived iPS cell line, clone \#3, a kind gift from Dr. James Thomson), were maintained on mouse embryonic fibroblasts (MEFs) (Globalstem Rockville, MD) in 6-well tissue culture plates (Thermo Fisher Scientific, Waltham, MA) with hESC medium: DMEM/F12 supplemented with glutamine, 20\% knockout serum replacement, non-essential amino acids, $0.1 \mathrm{mM} \beta$ mercaptoethanol (all from Invitrogen, Inc., Carlsbad, CA), to fibroblast growth factor 2 (FGF2) (20 ng/ml, Chemicon). For experiments using feeder-free conditions, human iPS cells were transitioned from fibroblast feeders to hESCqualified matrigel-coated galvanotaxis chamber with small colony clusters. Cells were allowed to grow in mTeSR ${ }^{\mathrm{TM}}$ maintenance medium (Stem Cell Technologies, Vancouver, $\mathrm{BC}$, Canada) for approximately $12 \mathrm{~h}$ at $37^{\circ} \mathrm{C}$ with $5 \% \mathrm{CO}_{2}$ prior to experiments. For ROCK inhibition experiments, Y27632 (Tocris Bioscience, Ellisville, MO) was added to the cells $30 \mathrm{~min}$ before exposure to an EF with the final concentration of $2 \mu \mathrm{M}$. The foreskin-derived fibroblast (CRL-2522 ${ }^{\mathrm{TM}}$, ATCC, Manassas, VA) was used as the control for hiPS cells.

Human embryonic stem cell line H7 (WiCell Research Institute, Madison, WI) was cultured in mTeSR ${ }^{\mathrm{TM}}$ maintenance medium at $37^{\circ} \mathrm{C}$ with $5 \% \mathrm{CO}_{2}$ with daily medium change. One day prior to the EF experiments, hESCs were treated with $1 \mathrm{mg} / \mathrm{ml}$ dispase (Invitrogen, Carlsbad, CA) and manually detached with a cell scraper. Small colony clusters were then plated in the hESC-qualified matrigelcoated electrotactic chamber in $\mathrm{mTeSR}^{\mathrm{TM}}$ culture medium, at a variety of cell densities up to $3 \sim 5 \times 10^{3}$ cells $/ \mathrm{cm}^{2}$. Cells were allowed to attach and grow for approximately $12 \mathrm{~h}$ at $37^{\circ} \mathrm{C}$ with $5 \% \mathrm{CO}_{2}$ prior to experiments. 


\section{D Matrigel Culture}

For 3D culture of human iPS cells, a stepped dilution using a combination of hiPS cell-qualified matrigel and mTeSR $^{\mathrm{TM}}$ medium was tested to optimize the culture condition. One day prior to the EFs experiments, hiPS cells were treated with $1 \mathrm{mg} / \mathrm{ml}$ dispase (Invitrogen, Carlsbad, CA) for $8 \mathrm{~min}$ and manually detached with a cell scraper.Cells at density of $0.5 \sim 1.0 \times 10^{5} / \mathrm{ml}$ were transferred to a $0.5-\mathrm{ml}$ tube and mixed well with matrigel at a volume ratio of $1: 1$ by gently pipetting up and down to distribute the colonies. $20 \mu \mathrm{l}$ of matrigel and cells mixtures were then transfer to a custom designed 3D electrotactic chamber in $60-\mathrm{mm}$ dishes, and placed in an incubator for $20 \mathrm{~min}$ to allow matrigel to polymerize. Once gel was formed, 4.5 to $5.0 \mathrm{ml} \mathrm{mTeSR}{ }^{\mathrm{TM}}$ medium was added into the dishes. Cells were allowed to grow overnight at $37^{\circ} \mathrm{C}$ with $5 \% \mathrm{CO}_{2}$.

EFs Stimulation and Time Lapse Image Recording

Prior to EF exposure, $\mathrm{mTeSR}^{\mathrm{TM}}$ containing $25 \mathrm{mM}$ HEPES buffer was added to one well of the chamber and the culture medium was observed to fill the opposite well assuring that the chamber was not clogged. EFs were applied to cells through two silver electrodes immersed in Steinberg's solution-filled reservoirs that were connected to wells at each end of the galvanotaxis chamber by two agar bridges (2\% agar in Steinberg's solution). hiPS or hES cells were exposed to an EF with strength ranging from 30 to $200 \mathrm{mV} / \mathrm{mm}$ for $3 \sim 8 \mathrm{~h}$ at $37^{\circ} \mathrm{C}$. Field strengths were measured directly at the beginning and the end of the experiment. Phase contrast (for 2D) and DIC (for 3D) time lapse imaging was performed using Nikon Eclipse imaging system equipped with a $\mathrm{CO}_{2}$ and temperature control chamber. 3D scanning was performed at various $\mathrm{Z}$ focal planes across the whole thickness of matrigel containing hES or hiPS cells colonies. Data analysis was performed using the NIH Image J software.

\section{Quantitative Analysis of Cell Behavior}

Cell migration was analyzed as previously reported [36, 37]. We used the NIH Image J software to quantify migration directedness and trajectory speed by tracing the position of cell nuclei at frame intervals of $10 \mathrm{~min}$. The directedness of migration was assessed as cosine $\theta$, where $\theta$ is the angle between the EFs vector and a straight line connecting the start and end position of a cell. A cell moving directly along the field lines toward the anode would have a directedness of -1 ; a cell moving directly toward the cathode would have a directedness of 1 . A value close to 0 represents random migration. The cosine $\theta$ will range from -1 to +1 , and an average of all the individual cells yields the directedness value for a population of cells, giving an objective quantification of the direction of cell migration. The trajectory speed $(\mathrm{Tt} / \mathrm{T})$ is the total migration distance of cells per minute; $\mathrm{Dx} / \mathrm{T}$ is an $\mathrm{x}$-axis displacement speed, which represents the migration along the EF vector.

\section{Immunostaining}

hiPS cells were seeded onto chambered glass-bottom dishes (Thermo Fisher Scientific) that were pre-coated with matrigel. Before or after EF stimulation, cells were washed with phosphate buffered saline (PBS), fixed with $2 \%$ paraformaldehyde for $15 \mathrm{~min}$ at room temperature. After 3 washes with PBS, cells were blocked with $3 \%$ bovine serum albumin (BSA) (Sigma-Aldrich). Cells were then incubated with Alexa Fluor 568 phalloidin (Invitrogen, USA) for $20 \mathrm{~min}$ at room temperature or primary antibodies against monoclonal mouse antiSSEA-4 (Developmental Studies Hybridoma Bank, Iowa) at 1:50 dilution and rabbit anti-Oct-4 (Chemicon) at 1:500 dilution overnight at $4^{\circ} \mathrm{C}$. After washes with PBS, cells were incubated with Alexa Fluor conjugatesecondary antibodies $(5 \mu \mathrm{g} / \mathrm{ml}$; Invitrogen) for $30 \mathrm{~min}$ at $4^{\circ} \mathrm{C}$, stained with Hoechst $33342(2 \mu \mathrm{g} / \mathrm{ml}$; Invitrogen $)$ for $5 \mathrm{~min}$, mounted using Fluoro-Gel with Tris Buffer (Electron Microscopy Sciences, Hatfield, PA), and imaged using an Olympus BX51 fluorescence microscope (Olympus, Center Valley, PA).

\section{Statistical Analysis}

Data are expressed as mean \pm SEM. Statistical differences between groups were assessed with unpaired, two-tailed Student's $t$-test, with statistical significance set at $p<0.05$. Statistical analysis was performed using the software of SPSS 10.0.

\section{Results}

\section{EF-Directed Migration of hiPs Cells}

First of all we determined the migratory behavior of hiPS cells in small colonies of $20-150$ cells. The colonies were relatively uniform and abundant. The following description, unless otherwise indicated, was from these colonies of hiPS cells. In the absence of an EF, no obvious directional migration was observed (Fig. 1a and b; supplemental movie 1). hiPS cells migrated very little and in random directions, as shown by the migration trajectories of individual cells (Fig. 1c). In an EF, hiPS cells migrated 

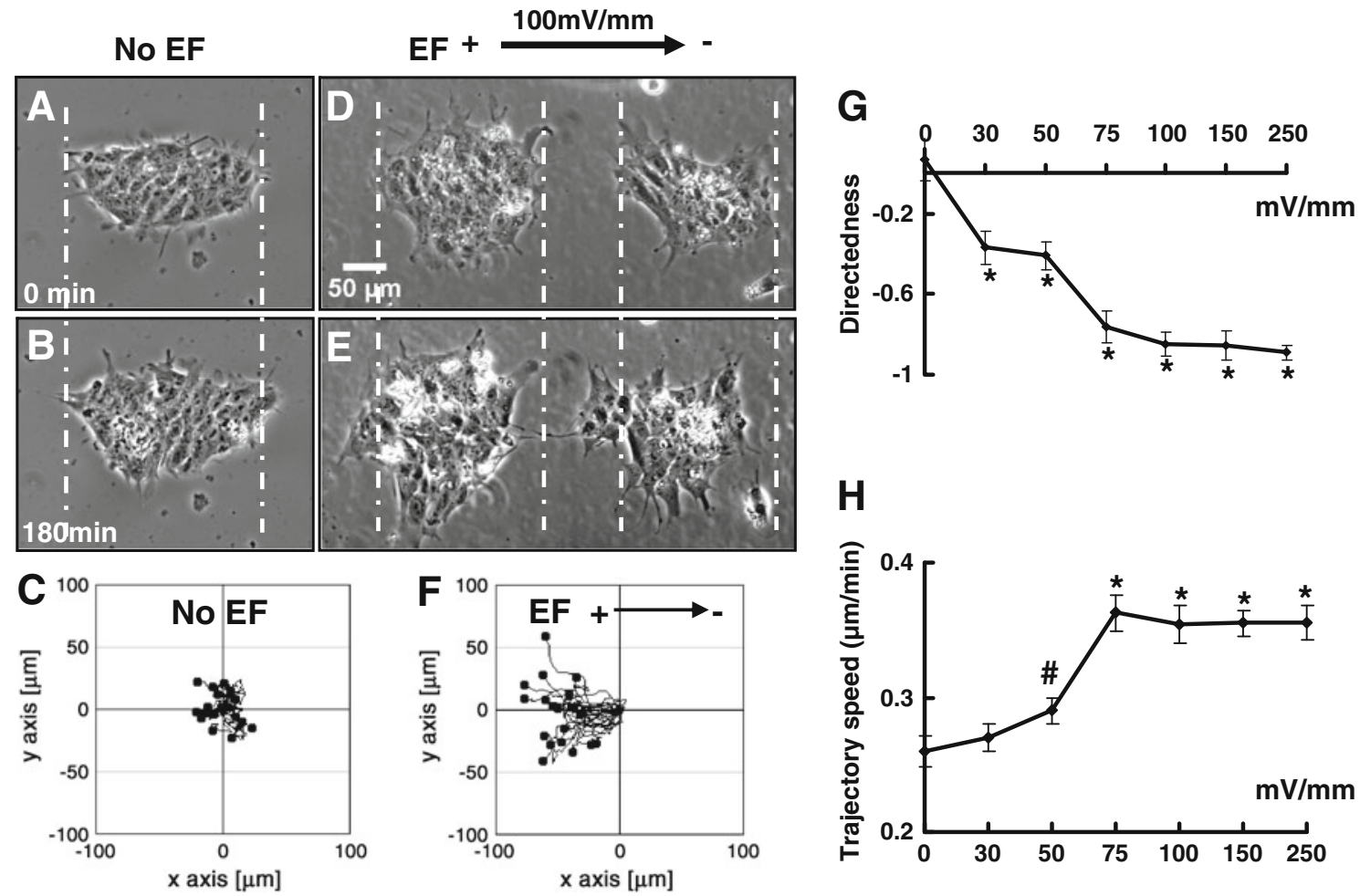

Fig. 1 Electric fields guided migration of hiPS cells in a voltage dependent manner. $\mathbf{a}$ and $\mathbf{b}$ In the absence of an EF, a hiPS cell colony showed no migration over a period of $3 \mathrm{~h}$. c Tracking of individual cells in the colony showed very little migration and any migration was in random directions. $\mathbf{d}$ and $\mathbf{e} \mathrm{An} \mathrm{EF}$ instigated and directed migration of the two colonies of hiPS cells toward the anode (left). $\mathbf{f}$ Tracking of individual cells showed the EF directed anodal migration, with increased motility. $\mathrm{EF}=100 \mathrm{mV} / \mathrm{mm}$; Scale bar, $50 \mu \mathrm{m}$. See Supplementary movies 1 and 2. g Migratory directedness of hiPS cells increased in a voltage dependent manner, with a threshold of $30 \mathrm{mV} / \mathrm{mm}$ or below. $\mathbf{h}$ EFs significantly increased the trajectory speed. \#, $P<0.05 ;{ }^{*}, P<0.01$ compared to the no EF control directionally toward the anode, at an increased speed (Fig. 1d, e and f; supplemental movie 2). Directional migration could be detected within $\sim 10$ min following the onset of the EF. Leading cells were seen to extend lamellipodia and filopodia toward the anode, while cells at the trailing edge were seen to retract extensions, and the whole colony migrated collectively. Cells largely maintained their relative position in the colony. We confirmed the EF-directed migration of hiPS cells by reversal of the EF polarity. Following reversal of the field polarity, hiPS cells changed their direction of migration and migrated toward the new anode (Fig. S1; supplemental movie 3).

To determine whether the density of hiPS cells affects EF-directed migration, we analyzed migration of hiPS cells in dissociated culture and culture of large colonies (>300 cells). Both the dissociated cells and the large colonies migrated toward the anode in an EF (Fig. S2; supplemental movie 4,5). Galvanotaxis of hiPS cells therefore remained very similar between dissociated cells and tightly connected cells in colonies, although their responses varied in terms of speed (dissociated cells: $0.50 \pm 0.02 \mu \mathrm{m} / \mathrm{min}$ vs. large colonies: $0.04 \pm$ $0.01 \mu \mathrm{m} / \mathrm{min}, p<0.05$ ) and directedness (dissociated cells: $-0.61 \pm 0.06$ vs. large colonies: $-0.96 \pm 0.01, p<$ $0.01)$. Cells in colonies migrated more directionally, and the larger the colony, the more uniformly they migrated toward the anode (Fig. 1f, S2 D, S2 H).

\section{Voltage-Dependent Galvanotaxis of hiPs Cells}

In order to determine the threshold voltage required to induce galvanotaxis, we analyzed the voltage-dependent galvanotaxis of hiPS cells. Significant directional migration was observed at a field strength of $30 \mathrm{mV} / \mathrm{mm}$ with directedness of $-0.37 \pm 0.08$ (Fig. 1g) $(p<0.01$, when compared with the no EF control). The threshold voltage required to induce galvanotaxis of hiPS cells was therefore $<30 \mathrm{mV} / \mathrm{mm}$. EFs of higher voltage significantly increased directedness, which doubled in an EF of $75 \mathrm{mV} / \mathrm{mm}$. Applied EFs also significantly increased trajectory speed. The increase in speed was dependent on field strength, with an EF of $75 \mathrm{mV} / \mathrm{mm}$ increasing the speed by $40 \%$ and this leveled out at higher voltages (Fig. 1h). Consistent with the directional migration, displacement along the $\chi$ axis (the field line toward the anode) in hiPS cells significantly increased following EF stimulation (Fig. S3). These results 
indicate that a robust galvanotaxis of hiPS cells occurs in vitro in an EF of $75 \mathrm{mV} / \mathrm{mm}$, under our experimental conditions, with a threshold of $<30 \mathrm{mV} / \mathrm{mm}$.

\section{Galvanotaxis of hiPs Cells in 3D Matrigel}

Next we tested whether hiPS cells would migrate in $3 \mathrm{D}$ culture and whether migration in $3 \mathrm{D}$ culture could be instigated and guided by applied EFs, as therapeutic use of EFs to guide cell migration will occur in a $3 \mathrm{D}$ environment. 3D electrotactic chambers were constructed, in which hiPS cells were cultured in 3D matrigel matrix. In the absence of an EF hiPS cells remained almost motionless over 5-8 h of observation in 3D culture (Fig. 2a and e). Directedness and trajectory speed were close to zero (Fig. 2c and d; supplemental movie 6,8). Application of an EF instigated the formation of lamellipodia and filopodia at the anodal side of colonies of hiPS cells in 3D culture, and induced significant directional migration toward the anode in a voltage-dependent manner, with a threshold of $<50 \mathrm{mV} / \mathrm{mm}$ (Fig. 2b, c, d and f). Both small and large colonies of hiPS cells extended polarized protrusions toward the anode and retracted protrusions facing the cathode (Fig. $2 \mathrm{~b}$ and f; supplemental movie 7,9). Cells migrated in the $\mathrm{Z}$ direction as well as toward the anode, therefore some cells gradually migrated out of focus. In large colonies, a group of leading cells formed, and the trailing cells migrated in lieu (Fig. 2f; supplemental movie 9). These results indicate that an EF can induce directional migration of otherwise stationary colonies of hiPS cells in a $3 \mathrm{D}$ environment. This may be of clinical significance as transplanted hiPS cells will reside in 3D tissues.

\section{EF Exposure Did Not Alter Expression of Stem Cell Markers in hiPs Cells}

In order to investigate whether EF exposure had any effect on the pluripotency of hiPS cells, we stained two stem cell markers, SSEA-4 and Oct-4, in hiPS cells following $5 \mathrm{~h}$ of EF exposure $(100 \mathrm{mV} / \mathrm{mm})$. Both SSEA-4 and Oct-4 were highly expressed in hiPS cells exposed to an EF (Fig. S4). The expression levels were similar to untreated control cells, indicating that EFs have no significant effect on the pluripotency of hiPS cells.

Galvanotaxis of hiPs Cells was Different from That of hES Cells

We compared the galvanotaxis of hiPS cells and hES cells because recent research has revealed differences in gene expression signatures between iPS cells and ES cells, although the pluripotency of hiPS cells and hES cells are thought to be similar [13]. Surprisingly, hES cells (H7) migrated toward the cathode, in the opposite direction of hiPS cells (Fig. 3a; supplemental movie 11 vs. supplemental movie 10). The migratory directedness of both hiPS cells and hES cells increased in a time-dependent manner. However, hiPS cells were more sensitive than hES cells and responded better. hiPS cells showed earlier galvanotaxis with directedness reaching a significant level $(-0.70 \pm 0.08)$ following $1 \mathrm{~h}$ in an EF, while hES cells took over $3 \mathrm{~h}$ to show significant directional migration, with lower directedness values $(0.14 \pm 0.12)$. The directedness of hES cells never reached a level comparable to that of hiPS cells (Fig. 3b). hES cells showed a higher trajectory speed than hiPS cells in an EF, although no difference was observed between the two cell types in the absence of EFs (Fig. 3c).

Rho/ROCK Signaling was Required for Galvanotaxis of hiPs Cells

To examine the underlying signaling mechanisms involved in the galvanotaxis of hiPS cells, we focused on the Rho/ ROCK signaling pathway, as Rho kinase (ROCK) inhibition has been used to promote the survival of iPS cells [14], and we have previously demonstrated its involvement in EF-directed migration [15]. hiPS cells were treated with Y$27632(2 \mu \mathrm{M})$, a specific inhibitor of ROCK, for $30 \mathrm{~min}$ prior to EFs application. EF-induced anodal migration of hiPS cells was significantly suppressed (Fig. 4b; supplemental movie 15 vs. supplemental movie 14). Quantitative analysis showed a sharp decline in migratory directedness from $-0.89 \pm 0.03$ in the no drug controls, to $-0.22 \pm 0.08$ in the Y-27632-treated hiPS cells, suggesting a critical role for Rho/ROCK signaling in the galvanotaxis of hiPS cells (Fig. 4e). Y-27632-treated cells showed increased motility both in the absence and presence of an EF (Fig. 4c and d; supplemental movie 13 vs. supplemental movie 12; supplemental movie 15 vs. supplemental movie 14). The trajectory speed of Y-27632-treated hiPS cells increased by $36 \%$ and $34 \%$ in the absence and presence of an EF respectively (Fig. 4f). Treatment with Y-27632 induced two distinct morphological changes: 1) loss of close cell-cell contacts; 2) multiple protrusions (Fig. $4 \mathrm{a}$ and b; Fig. S5; supplemental movies 13 and 15).

\section{Discussion}

With the aim of developing novel techniques to guide human stem cells, to facilitate stem cell therapy, we investigated the application of EFs to guide hiPS cell migration in both $2 \mathrm{D}$ and $3 \mathrm{D}$ cultures. We reported that: 1) a DC EF guided migration of hiPS cells in 2D culture; colonies showed significantly greater directionality than dissociated cells; 2) hiPS cells remained immotile in a 3D 
A
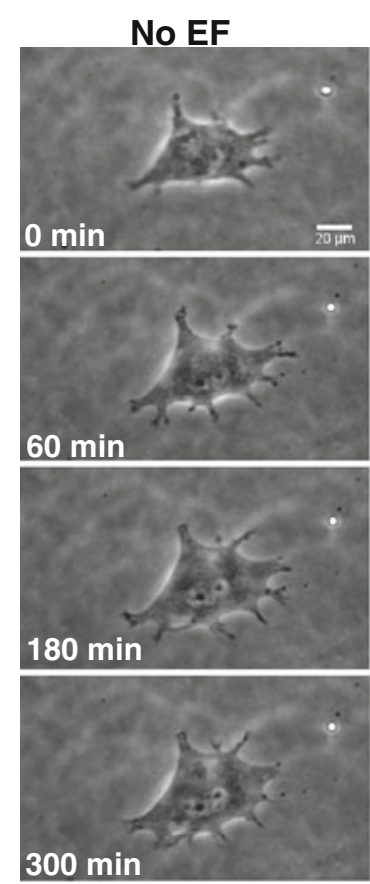

B
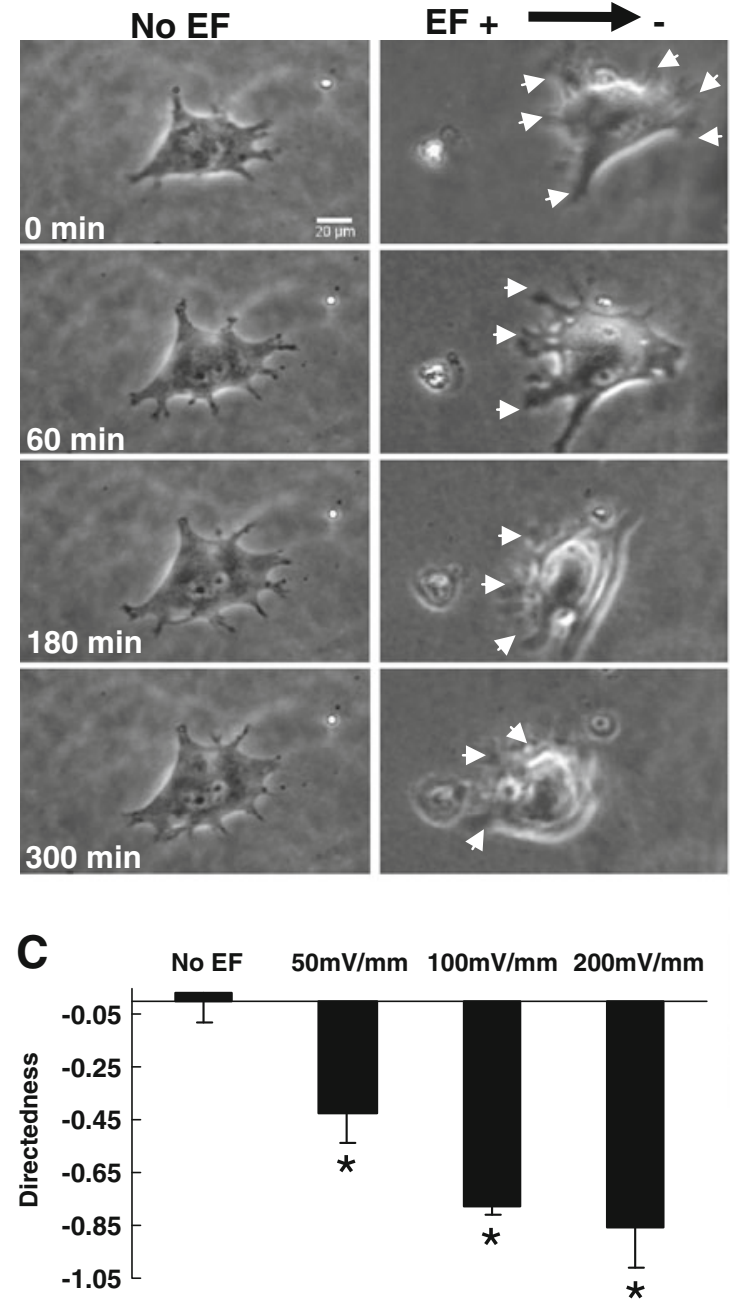

D

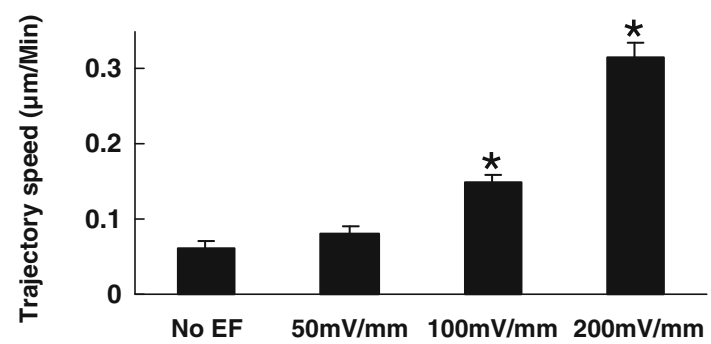

Fig. 2 EFs stimulated and guided migration of hiPS cells in 3D matrigel. a and e Small and large colonies of hiPS cells remained immotile in control culture without an EF over 5 and $8 \mathrm{~h}$, respectively. b A small colony extended multiple protrusions to the anode and migrated in the same direction. f A large colony enlarged a leading process toward the anode and the whole colony migrated to the anode
$\mathbf{E}$

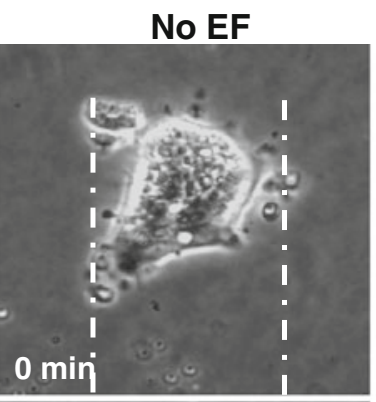

$\mathbf{F}$
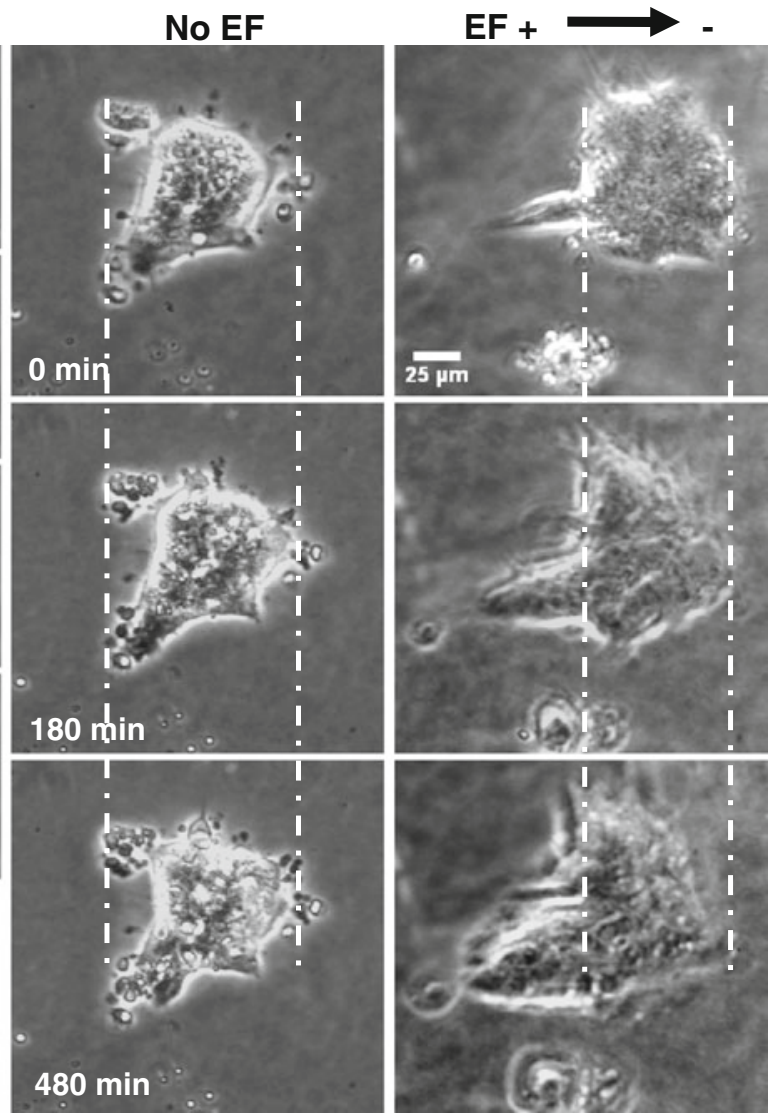

(left). Scale bar, $20 \mu \mathrm{m}$ in A and B; $25 \mu \mathrm{m}$ in E and F. See Supplementary movies $6,7,8$ and 9 . c and $\mathbf{d}$ EFs significantly increased trajectory speed (C) and migratory directedness (D) of hiPS cells in 3D matrigel in a voltage-dependent manner, with a threshold of $<50 \mathrm{mV} / \mathrm{mm}$. ${ }^{*}, P<0.01$ compared to the no EF control

did not affect the expression of stem cell markers; 5) hiPS cells migrated toward the anode with greater sensitivity and directedness compared to hES cells which migrated toward the cathode; 6) ROCK inhibition by Y-27632 significantly 

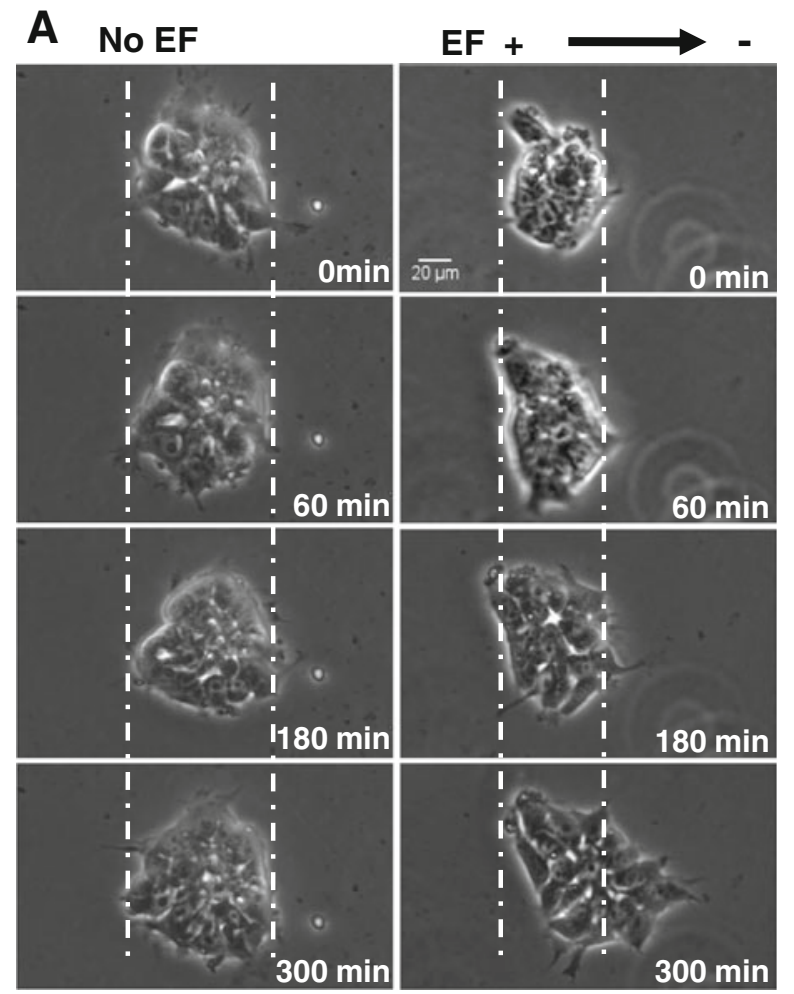

B

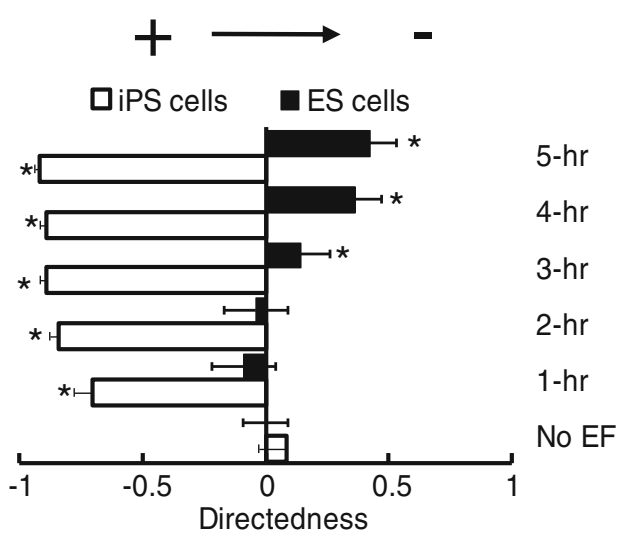

C

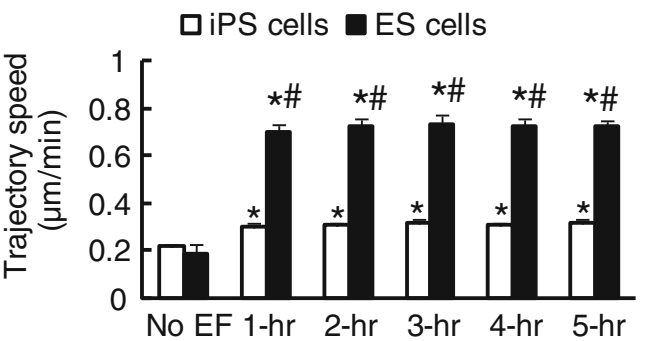

Fig. 3 Galvanotaxis of hiPS cells was different from that of hES cells. a No migration (left panel) of a colony of hES (H7) cells occurred in the absence of an EF. Right panel showed directional migration toward the cathode (right) of a hES cell colony in the presence of an EF. Scale bar, $20 \mu \mathrm{m}$. b hiPS cells and hES cells migrated in opposite directions in an EF. hiPS cells migrated toward the anode, whereas hES cells migrated toward the cathode. hiPS cells manifested robust galvanotaxis with directedness of $-0.70 \pm 0.08$ within $1 \mathrm{~h}$ in an $\mathrm{EF}$, whereas in the same field, hES cells presented a directeness value of only $0.14 \pm 0.12$ after $3 \mathrm{~h}$. c EFs significantly increased the trajectory speed in both hiPS and hES cells. ${ }^{*}, P<0.01$ when compared to the no EF control; \#, $P<0.01$ compared to hiPS cells. $\mathrm{EF}=100 \mathrm{mV} / \mathrm{mm}$. See Supplementary movies 10 and 11

of many types of cells. We have recently provided evidence that EFs could be a predominant guidance cue for corneal epithelial wound healing, over-riding contact inhibition release, free edge, injury stimulation and other wellaccepted guidance cues [12].

There are several important points to consider when developing methods of electrical stimulation and guidance of hiPS cells for possible practical use. First is the threshold of applied EFs. 3D tissues are volume conductors in which EFs dissipate exponentially. To maintain a constant highvoltage gradient in electrically conductive tissue inevitably involves issues such as electrode products, heat generation, effects on other types of cells, and permeability of blood vessels. Low voltage offers many advantages. In our experiments, an EF of $30 \mathrm{mV} / \mathrm{mm}$ induced significant directional migration. This is a voltage below the thresholds for galvanotaxis of fibroblasts and endothelial cells $[23,24]$. Application of an EF of this strength may therefore selectively guide migration of hiPS cells without significant effects on fibroblasts and endothelial cells. The second point is the migration of clusters of cells. Large and damaged tissue [20-22]. Applied EFs guide migration 
A
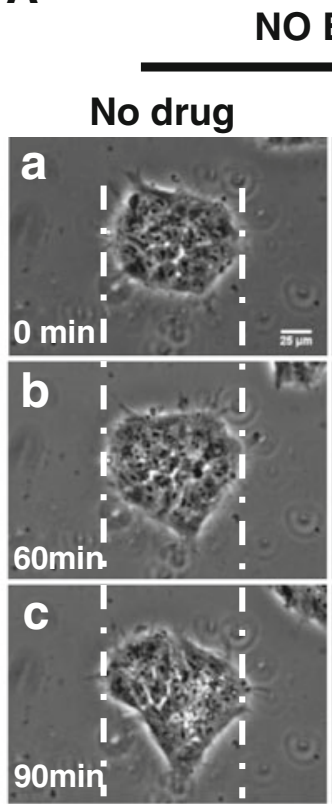

$+Y-27632$
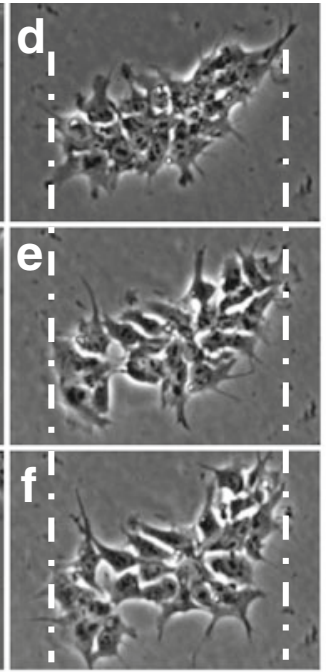

C

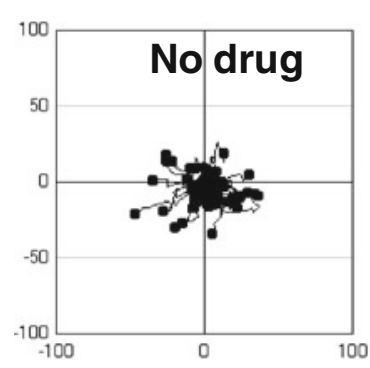

NO EF

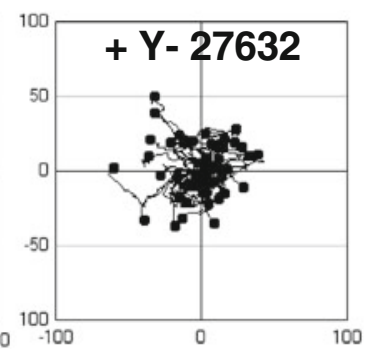

$\mathbf{E}$

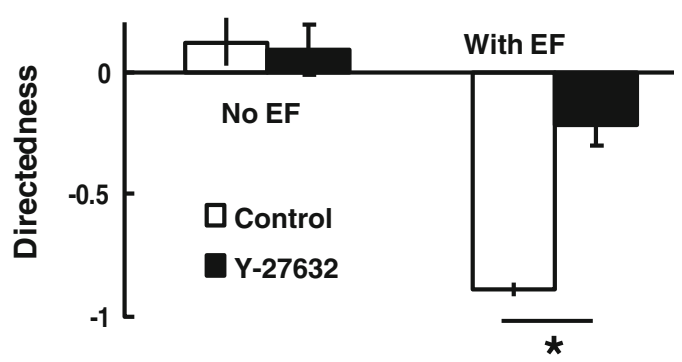

Fig. 4 Rho A/ROCK signalling required for galvanotaxis of hiPS cells. a and b Y-27632, a ROCK inhibitor, induced a tendency for dissociation of cells from the colony to inhibited galvanotaxis. $\mathbf{c}$ and $\mathbf{d}$ Inhibition of Rock by Y-27632 increased the motility of hiPS cells and inhibited the directional migration. $\mathbf{e}$ and $\mathbf{f}$ Quantitative analysis

numbers of hiPS cells will need to be used for transplantation. Clusters of cells are normally immotile, although some cells may move within the clusters (Figs. 1a, 2a and e). This poses a problem for the transplanted cells to integrate
B

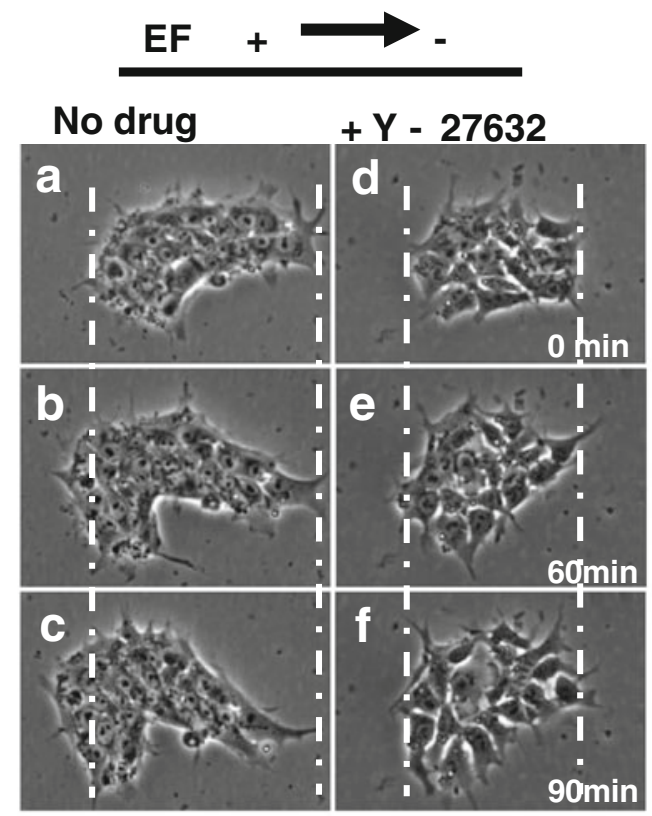

D



$\mathbf{F}$

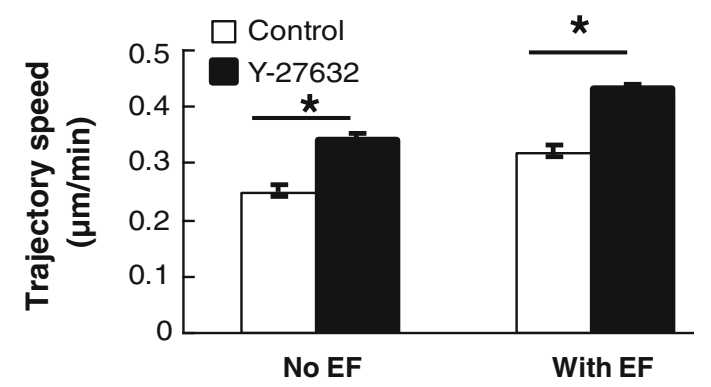

showed a sharp decline in migratory directedness and a significant increase in the trajectory speed in Y-27632-treated hiPS cells. Scale bar, $25 \mu \mathrm{m}$. EF $=100 \mathrm{mV} / \mathrm{mm}$. See Supplementary movies 12, 13, 14 and 15

with the host tissue. Very encouragingly, we have shown that an applied EF was able to stimulate and induce directional migration of clusters of hiPS cells. Thirdly, transplanted cells have to migrate in a $3 \mathrm{D}$ environment. We have shown that 
hiPS cells did not migrate in 3D in matrigel in the absence of an EF (Fig. 2a and e). Excitingly, applied EFs stimulated and guided migration of hiPS cells. Exogenously applied EFs have been safely used in the treatment of chronic wounds, neurorehabilitation, and spinal cord injury [25-27]. Our results thus provide a basis for developing stimulation techniques for guiding migration of transplanted hiPS cells in vivo where they need to migrate in $3 \mathrm{D}$.

There are a number of important differences in galvanotaxis between hiPS cells and hES cells, although the biological potency and epigenetic state of iPS cells are indistinguishable from those of ES cells [28]. Surprisingly, hES (H7) cells migrated in a direction opposite to that of hiPS cells in an EF; hES cells migrated toward the cathode (Fig. 3). Since cathodal migration is the most popular migratory response in many different types of cells, we employed the foreskin dermal fibroblasts, from which the hiPS cells were generated in this study, as the cell control, and found that the nonprogrammed fibroblast also migrate toward the anode in EFs, same to that observed in hiPS cells (supplemental figures 6 and 7; supplemental movies 16, 17, 18 and 19). In addition, $\mathrm{hES}$ cells were less sensitive to an EF. The same voltage (100 $\mathrm{mV} / \mathrm{mm})$ took 3-4 times longer time to induce a directional response in hES cells and the response was much weaker (Fig. 3b). Thus, these results revealed new differences in cellular behavior between iPS and ES cells. Whether these differences are due to the different gene expression signatures will need to be studied [13]. Nevertheless, electrical stimulation might be particularly suitable in therapies using iPS cells compared to ES cells.

ROCK is known to be important to maintain iPS and ES cell cultures and we found that it critically influenced galvanotaxis of hiPS cells. ROCK is the downstream effector kinase of Rho A, a family member of the Rho GTPases. Rho A/ROCK signaling has been implicated in cytoskeleton remodeling, cell adhesion, membrane protrusion, and cell migration [29-31]. Recent studies demonstrated that inhibition of ROCK with Y27632 was an effective means of increasing the survival rate of stem cells including hES and hiPS cells [14, 32]. The ROCK inhibitor therefore is considered as a promising molecule to aid in the expansion of pluripotent stem cells for regenerative medicine [33]. We and others have previously found an important role for ROCK in galvanotaxis [15, 34]. Y-27632 at $2 \mu \mathrm{M}$, a concentration much lower than that normally used for enhancing the survival rate of stem cells, dramatically decreased the directedness of hiPS cells in an EF by $75 \%$. ROCK activation thus is involved in galvanotaxis of hiPS cells. A significant decline in directional migration following ROCK inhibition was associated with an increase in the migratory speed of hiPS cells (Fig. 4e and f). Reduced cell-cell contact and increased filopodium formation in hiPS cells also occurred following ROCK inhibition in hiPS cells (Fig. 4a and b, Fig. S5). Our results are consistent with a previous report suggesting ROCK inhibition promoted cell motility in mouse ES cells [35]. When considering guiding stem cell migration electrically, the effect of ROCK inhibition will be an important factor.

In summary, a very small EF $(30 \mathrm{mV} / \mathrm{mm})$ is an effective cue to guide and stimulate migration of hiPS cells. Most importantly, applied EFs can direct migration of large clusters of hiPS cells which are normally stationary in a 3D environment. This response depends on Rho/ROCK signaling. The size, duration and direction of applied EFs can be relatively easily controlled. Electrical stimulation may offer a practical approach to facilitate stem cell therapy, where guided cell migration and integration with host tissue are needed. These results may lead to techniques for applying EFs in vivo to guide migration of transplanted stem cells.

Acknowledgements This work is supported by a grant from the California Institute of Regenerative Medicine RB1-01417 (to MZ). MZ is also supported by NIH 1R01EY019101, NSF MCB-0951199, and the UC Davis Dermatology Developmental Fund. WD is in part supported by grants from the National Institutes of Health (RO1 NS059043 and RO1 ES015988), National Multiple Sclerosis Society, Feldstein Medical Foundation, and Shriners Hospitals for Children. We thank James Thomson for providing iPS cells and Jan Nolta for her advice and support. We also thank Bing Song for sharing technical expertise, and other members from the Zhao, Deng to Li labs for their help.

Author contribution $\mathrm{MZ}$ and $\mathrm{YH}$ designed the research; JZ, MC, $\mathrm{QZ}$ and XZ performed the research; WD and RL contributed reagents and cell lines; JZ and MZ analyzed the data; JZ and MZ wrote the paper with help from WD to XZ.

\section{Conflicts of interest None}

Open Access This article is distributed under the terms of the Creative Commons Attribution Noncommercial License which permits any noncommercial use, distribution, and reproduction in any medium, provided the original author(s) and source are credited.

\section{References}

1. Shi, Y. (2009). Induced pluripotent stem cells, new tools for drug discovery and new hope for stem cell therapies. Current Molecular Pharmacology, 2, 15-18.

2. Smart, N., \& Riley, P. R. (2008). The stem cell movement. Circulation Research, 102, 1155-1168.

3. Okano, H., \& Sawamoto, K. (2008). Neural stem cells: Involvement in adult neurogenesis and CNS repair. Philosophical Transactions of the Royal Society of London. Series B: Biological Sciences, 363, 2111-2122.

4. Burst, V. R., et al. Poor cell survival limits the beneficial impact of mesenchymal stem cell transplantation on acute kidney injury. Nephron Experimental Nephrology, 114, e107-116.

5. Dai, W., et al. (2005). Allogeneic mesenchymal stem cell transplantation in postinfarcted rat myocardium: Short- and longterm effects. Circulation, 112, 214-223. 
6. Hayakawa, J., et al. (2009). Dextran sulfate and stromal cell derived factor-1 promote CXCR4 expression and improve bone marrow homing efficiency of infused hematopoietic stem cells. Journal of Nippon Medical School, 76, 198-208.

7. Kelly, S., et al. (2004). Transplanted human fetal neural stem cells survive, migrate, and differentiate in ischemic rat cerebral cortex. Proceedings of the National Academy of Sciences of the United States of America, 101, 11839-11844.

8. Miller, R. J., Banisadr, G., \& Bhattacharyya, B. J. (2008). CXCR4 signaling in the regulation of stem cell migration and development. Journal of Neuroimmunology, 198, 31-38.

9. Sackstein, R., et al. (2008). Ex vivo glycan engineering of CD44 programs human multipotent mesenchymal stromal cell trafficking to bone. Natural Medicines, 14, 181-187.

10. Maeng, Y. S., et al. (2009). Endothelial progenitor cell homing: Prominent role of the IGF2-IGF2R-PLCbeta2 axis. Blood, 113, 233-243.

11. Christopherson, K. W., 2nd, Hangoc, G., Mantel, C. R., \& Broxmeyer, H. E. (2004). Modulation of hematopoietic stem cell homing and engraftment by CD26. Science, 305, 1000-1003.

12. Zhao, M. (2009). Electrical fields in wound healing-An overriding signal that directs cell migration. Seminars in Cell \& Developmental Biology, 20, 674-682.

13. Chin, M. H., et al. (2009). Induced pluripotent stem cells and embryonic stem cells are distinguished by gene expression signatures. Cell Stem Cell, 5, 111-123.

14. Claassen, D. A., Desler, M. M., \& Rizzino, A. (2009). ROCK inhibition enhances the recovery and growth of cryopreserved human embryonic stem cells and human induced pluripotent stem cells. Molecular Reproduction and Development, 76(8), 722-732.

15. Yao, L., Shanley, L., McCaig, C., \& Zhao, M. (2008). Small applied electric fields guide migration of hippocampal neurons. Journal of Cellular Physiology, 216, 527-535.

16. Li, Q., et al. (2009). Transplantation of MSCs in combination with netrin-1 improves neoangiogenesis in a rat model of hind limb ischemia. Journal of Surgical Research, [Epub ahead of print]

17. Aguirre, A., Rizvi, T. A., Ratner, N., \& Gallo, V. (2005). Overexpression of the epidermal growth factor receptor confers migratory properties to nonmigratory postnatal neural progenitors. The Journal of Neuroscience, 25, 11092-11106.

18. Suzuki, T., Mandai, M., Akimoto, M., Yoshimura, N., \& Takahashi, M. (2006). The simultaneous treatment of MMP-2 stimulants in retinal transplantation enhances grafted cell migration into the host retina. Stem Cells, 24, 2406-2411.

19. Song, M., et al. Using a neodymium magnet to target delivery of ferumoxide-labeled human neural stem cells in a rat model of focal cerebral ischemia. Human Gene Therapy, 21, 603-610

20. McCaig, C. D., Rajnicek, A. M., Song, B., \& Zhao, M. (2005). Controlling cell behavior electrically: Current views and future potential. Physiological Reviews, 85, 943-978.

21. Reid, B., Nuccitelli, R., \& Zhao, M. (2007). Non-invasive measurement of bioelectric currents with a vibrating probe. Nature Protocols, 2, 661-669.
22. Nuccitelli, R., Nuccitelli, P., Ramlatchan, S., Sanger, R., \& Smith, P. J. (2008). Imaging the electric field associated with mouse and human skin wounds. Wound Repair and Regeneration, 16(3), $432-441$.

23. Zhao, M., Bai, H., Wang, E., Forrester, J. V., \& McCaig, C. D. (2004). Electrical stimulation directly induces pre-angiogenic responses in vascular endothelial cells by signaling through VEGF receptors. Journal of Cell Science, 117, 397-405.

24. Guo, A., et al. (2010). Effects of physiological electric fields on migration of human dermal fibroblasts. Journal of Investigative Dermatology, [Epub ahead of print]

25. Gardner, S. E., Frantz, R. A., \& Schmidt, F. L. (1999). Effect of electrical stimulation on chronic wound healing: A meta-analysis. Wound Repair and Regeneration, 7, 495-503.

26. Shapiro, S., et al. (2005). Oscillating field stimulation for complete spinal cord injury in humans: A phase 1 trial. Journal of Neurosurgery Spine, 2, 3-10.

27. Harris-Love, M. L., \& Cohen, L. G. (2006). Noninvasive cortical stimulation in neurorehabilitation: A review. Archives of Physical Medicine and Rehabilitation, 87, S84-S93.

28. Wernig, M., et al. (2007). In vitro reprogramming of fibroblasts into a pluripotent ES-cell-like state. Nature, 448, 318-324.

29. Woods, A., Wang, G., \& Beier, F. (2005). RhoA/ROCK signaling regulates Sox9 expression and actin organization during chondrogenesis. The Journal of Biological Chemistry, 280, 11626-11634.

30. Totsukawa, G., et al. (2004). Distinct roles of MLCK and ROCK in the regulation of membrane protrusions and focal adhesion dynamics during cell migration of fibroblasts. The Journal of Cell Biology, 164, 427-439.

31. Zohrabian, V. M., Forzani, B., Chau, Z., Murali, R., \& JhanwarUniyal, M. (2009). Rho/ROCK and MAPK signaling pathways are involved in glioblastoma cell migration and proliferation. Anticancer Research, 29, 119-123.

32. Watanabe, K., et al. (2007). A ROCK inhibitor permits survival of dissociated human embryonic stem cells. Nature Biotechnology, $25,681-686$.

33. Gauthaman, K., Fong, C. Y., \& Bongso, A. (2010). Effect of ROCK inhibitor Y-27632 on normal and variant human embryonic stem cells (hESCs) in vitro: Its benefits in hESC expansion. Stem Cell Reviews, 6, 86-95.

34. Rajnicek, A. M., Foubister, L. E., \& McCaig, C. D. (2006). Temporally and spatially coordinated roles for Rho, Rac, Cdc42 and their effectors in growth cone guidance by a physiological electric field. Journal of Cell Science, 119, 1723-1735.

35. Harb, N., Archer, T. K., \& Sato, N. (2008). The Rho-RockMyosin signaling axis determines cell-cell integrity of selfrenewing pluripotent stem cells. PLoS ONE, 3, e3001.

36. Zhao, M., et al. (2006). Electrical signals control wound healing through phosphatidylinositol-3-OH kinase-gamma and PTEN. Nature, 442, 457-460.

37. Tai, G., Reid, B., Cao, L., \& Zhao, M. (2009). Electrotaxis and wound healing: Experimental methods to study electric fields as a directional signal for cell migration. Methods in Molecular Biology, 571, 77-97. 